\title{
Monitoramento In Situ e em Tempo Real de Variáveis Morfológicas do Poli(cloreto de vinila) Usando Espectroscopia NIR
}

João M. de Faria Jr. Braskem S.A.

\author{
Enrique L. Lima, José C. Pinto \\ Programa de Engenharia Química, COPPE, UFRJ \\ Fabricio Machado \\ Instituto de Química, UnB
}

\begin{abstract}
Resumo: Este trabalho ilustra o uso de espectroscopia do infravermelho próximo (NIRS) para fins de monitoramento da polimerização em suspensão de cloreto de vinila em tempo real. Resultados inéditos mostraram que é possível acompanhar a evolução de importantes propriedades morfológicas de resinas de PVC [poli(cloreto de vinila)], como por exemplo, BD (densidade aparente), CPA (absorção de plastificante a frio), DTP (distribuição de tamanho de partículas) e Dp (diâmetro de partícula). Mostrou-se também, pela primeira vez, que é possível analisar quantitativamente, com o auxílio da sonda NIRS in situ, a estrutura morfológica da partícula de PVC. Com a possibilidade de predizer a evolução dinâmica dos parâmetros morfológicos em tempo real, mostrou-se que é possível injetar dispersantes e variar a velocidade de agitação durante a reação para fins de controle das variáveis BD, CPA e DTP do PVC, até que o ponto de identificação da partícula seja atingido. Por meio do monitoramento e da estratégia de controle proposta, é possível antecipar fugas de temperatura no reator, aumentar a segurança do processo, diminuir o tempo de desenvolvimento de resinas com características morfológicas diferenciadas, evitando perdas de margem oriundas da venda de produtos fora de especificação, e otimizar os recursos para o desenvolvimento de novos produtos.
\end{abstract}

Palavras-chave: Poli(cloreto de vinila), espectroscopia NIR, polimerização em suspensão, propriedades morfológicas.

\section{Monitoring In Situ and in Real Time the Morphological Variables of PVC Using NIR Spectroscopy}

Abstract: This work illustrates the use of near infrared spectroscopy (NIRS) for monitoring of the suspension polymerization of vinyl chloride in real time. Obtained results showed that it is possible to track in situ and in real time important morphological properties of PVC resins, such as BD (bulk density), CPA (cold plasticizer absorption), PSD (particle size distribution) and Dp (average particle diameter). It was also shown for the first time that it is possible to analyze quantitatively, with the help of in situ NIRS probe, the morphological structure of the PVC particles. As a consequence, it was shown that is possible to inject dispersants and vary the agitation speed during the reaction for proper control of BD, CPA and the PSD of PVC, before attainment of the particle identification point. Through the proposed monitoring and control strategies, it is possible to anticipate reactor disturbances (increasing the process safety) and to reduce the time needed for development of resins with different morphological characteristics (avoiding losses caused by off-specification), allowing for optimization of the product development process.

Keywords: Poly(vinyl chloride), NIR spectroscopy, suspension polymerization, morphological properties.

\section{Introdução}

As indústrias de polimerização trabalham com a idéia de aumentar a produtividade de produtos de alta qualidade, com o menor custo possível e empregando processos mais flexíveis e seguros. Isto é fortemente motivado pela competição comercial agressiva, pelo aumento das expectativas de qualidade dos clientes e pela pressão da sociedade por processos e plantas menos poluentes e ambientalmente cor- retas. Estas mudanças podem ser enfrentadas mais eficientemente com a ajuda de modelagem e técnicas de controle e otimização do processo ${ }^{[1]}$.

Resinas poliméricas com propriedades especiais são freqüientemente exigidas para uma dada aplicação. Para alcançar a produção de uma resina polimérica com propriedades finais especificadas, a disponibilidade de técnicas de controle eficientes pode não ser suficiente. A distância existen-

Autor para correspondência: José C. Pinto, Programa de Engenharia Química, COPPE, UFRJ, Cidade Universitária, Caixa Postal 68502, CEP: 21945-970, Rio de Janeiro, RJ, Brasil. E-mail: pinto@peq.coppe.ufrj.br 
te entre o processo de polimerização e a técnica de controle deve ser reduzida com instrumentação acurada e robusta para monitoramento em linha, de maneira que o controlador possa ser alimentado corretamente com o estado do processo real. A falta de instrumentos que possam ser acoplados diretamente para medir e monitorar a qualidade da resina polimérica é reconhecido como o problema mais crítico no campo do controle de reatores de polimerização ${ }^{[2]}$.

Os principais fatores que limitam o desenvolvimento de estratégias para controlar as propriedades do polímero incluem a disponibilidade limitada e o custo da instrumentação em linha, a falta de um entendimento detalhado da dinâmica do processo e o comportamento não linear do processo, altamente sensível a perturbações. Tecnologias de controle de processos apropriadas e técnicas de otimização dão subsídios para reduzir custos e melhorar a consistência do produto, por meio da capacitação do processo para operar o mais próximo das restrições econômicas, de planta e de segurança $\mathrm{a}^{[3]}$. Além disso, é sabido que durante a produção industrial de polímeros ocorrem perdas consideráveis por falhas e/ou flutuação das condições operacionais, muitas das vezes identificadas após algumas horas do término do ciclo de reação. Isto é particularmente verdade para os processos em batelada. Neste cenário, a técnica de espectroscopia de infravermelho próximo (NIRS) oferece algumas oportunidades para monitoramento e controle das características morfológicas da resina de PVC in situ e em tempo real, como mostrado neste trabalho.

Neste trabalho é ilustrado o uso de espectroscopia do infravermelho próximo para fins de monitoramento em tempo real da polimerização em suspensão de cloreto de vinila, buscando a predição e o acompanhamento de importantes propriedades morfológicas do PVC, como por exemplo, BD, CPA, DTP e Dp.

\section{Experimental}

Uma receita padrão consistindo de álcool polivinílico, iniciador, água e monômero foi alimentada ao reator para realização das reações de polimerização. A receita detalhada não será disponibilizada por questões de confidencialidade. O aparato de reação polimérica é o mesmo utilizado por de Faria $\mathrm{Jr}^{[1]}$, e o leitor pode recorrer a esta referência para obter uma descrição mais detalhada. Os estudos foram divididos em cinco grupos principais.

Os testes do primeiro grupo de estudos buscaram determinar a sensibilidade dos espectros de infravermelho próximo a mudanças das características físicas e físico-químicas de resinas industriais de PVC e foram conduzidos tomando como base quatro resinas comerciais da Braskem. Antes de conduzir os estudos de sensibilidade, esses diferentes produtos foram analisados visando a identificar suas características principais (massas molares médias, umidade e CPA) e determinar as eventuais diferenças existentes.

O segundo grupo de estudos consistiu em realizar reações de polimerização do MVC (monocloreto de vinila) em suspensão, em diferentes condições de operação, para obtenção de PVC, sem o monitoramento por sonda NIR. Os testes foram organizados segundo um plano fatorial completo a 2 níveis para 2 variáveis independentes (temperatura e concentração de iniciador) e em tréplicas. Foram também realizadas 10 réplicas da condição central. As duas variáveis independentes escolhidas estão associadas a estratégias de operação normalmente usadas industrialmente. A temperatura é utilizada para controlar o valor $\mathrm{K}$ da resina (medida indireta da massa molar), enquanto a concentração de iniciador é utilizada para controlar a velocidade da reação, e conseqüentemente definir a política de geração de calor (troca térmica). A condição central foi utilizada para avaliação dos erros experimentais.

O terceiro grupo de estudos foi executado para obter a evolução dinâmica da densidade aparente (BD) e da absorção de plastificante a frio (CPA).

O quarto grupo de estudos foi conduzido para verificar se as mudanças na velocidade da agitação resultariam em modificações da BD, do CPA e do diâmetro médio de partículas finais do PVC. Os testes foram executados em reatores bancada de aço inox com volume entre 0,001 e $0,01 \mathrm{~m}^{3}$, com pressão e temperatura máxima de trabalho de 20 bar e $250{ }^{\circ} \mathrm{C}$, respectivamente. Um banho termostático foi usado para controlar a temperatura da reação. A velocidade da agitação poderia ser variada na faixa de 0 a $2400 \mathrm{rpm}$ e um termopar tipo Pt 100 foi usado para acompanhamento e controle da temperatura do reator. Após 20 minutos de reação, a velocidade normalizada da agitação foi mudada na escala de 0,40 a 1,00. Em todos os grupos a velocidade normalizada de agitação no início da reação era igual a 0,6 . As corridas foram replicadas para analisar a reprodutibilidade do processo. Para cada corrida, a BD, o CPA e a DTP do pó final do PVC foram obtidos.

O quinto grupo de estudos foi executado dosando o agente de suspensão ao meio reacional a fim verificar se as mudanças da concentração do agente de suspensão conduziriam às modificações da $\mathrm{BD}$, do $\mathrm{CPA}$ e do diâmetro médio de partículas finais do PVC, como nos testes precedentes. Os testes foram executados em um reator de aço inox piloto com volume na faixa de $0,1 \mathrm{~m}^{3}$ a $1 \mathrm{~m}^{3}$. Um controle em cascata (PID-PI) combinado com um sistema de controle distribuído foi usado para controlar a vazão de água quente na camisa do reator. Após 20 minutos da reação, a quantidade normalizada de agente de suspensão foi dosada no reator $(0,58 ; 0,75$; $0,83 ; 1,00)$. As adições foram executadas em diferentes bateladas, usando uma solução de agente dispersante em água. Esta solução foi mantida dentro de um vaso de processo que era isolado do meio da reação por uma válvula manual. Após 20 minutos de reação, a válvula foi aberta, permitindo que a solução fluísse para o reator. 


\section{Resultados e Discussão}

Foram identificadas diferenças significativas de morfologia e estrutura molecular entre os diferentes grades industriais analisados como mostrado na Tabela 1.

Os cromatogramas obtidos para cada resina são apresentados na Figura 1. Os resultados indicaram que a distribuição de massas molares segue em todos os casos a distribuição clássica de Schultz-Flory, com índice de polidispersão igual a 2, controlado pelo modo de transferência de cadeia para o monômero.

Analisando os espectros mostrados na Figura 2, é possível observar que as análises de infravermelho próximo indicaram que as medidas são estáveis, pois não são sensíveis a mudanças nos níveis de agitação. Este resultado é muito importante pois não há mudanças de qualquer tipo nas resinas com o aumento do nível de agitação. Observa-se também que há diferenças entre os diferentes grades industriais analisados, embora o efeito da carga total de material orgânico tenha sido o mais pronunciado.

Os resultados obtidos mostram que os espectros de NIR de diferentes grades são diferentes, o que estimula o desenvolvimento de estratégias de controle da morfologia final da partícula (controle da coalescência) baseado em espectroscopia do infravermelho próximo, a partir de ensaios reais de polimerização. É importante enfatizar que os testes preliminares não permitiram identificar a verdadeira fonte das diferenças detectadas no NIR.

A análise do plano fatorial do segundo grupo de estudo mostrou que o tempo de reação é influenciado pela concen-

Tabela 1. Massas molares médias normalizadas, absorção de platificantes a frio e percentual de umidade das amostras.

\begin{tabular}{cccccc}
\hline Amostra & Umidade (\%) & CPA (pcr) & Mw & Mn & IP \\
\hline Resina A & 0,35 & mínimo 30 & 1,00 & 0,48 & 2,08 \\
Resina B & 0,32 & mínimo 25 & 0,81 & 0,40 & 2,01 \\
Resina C & 0,27 & - & 0,78 & 0,39 & 1,98 \\
Resina D & 0,23 & mínimo 16 & 0,52 & 0,27 & 1,89 \\
\hline
\end{tabular}

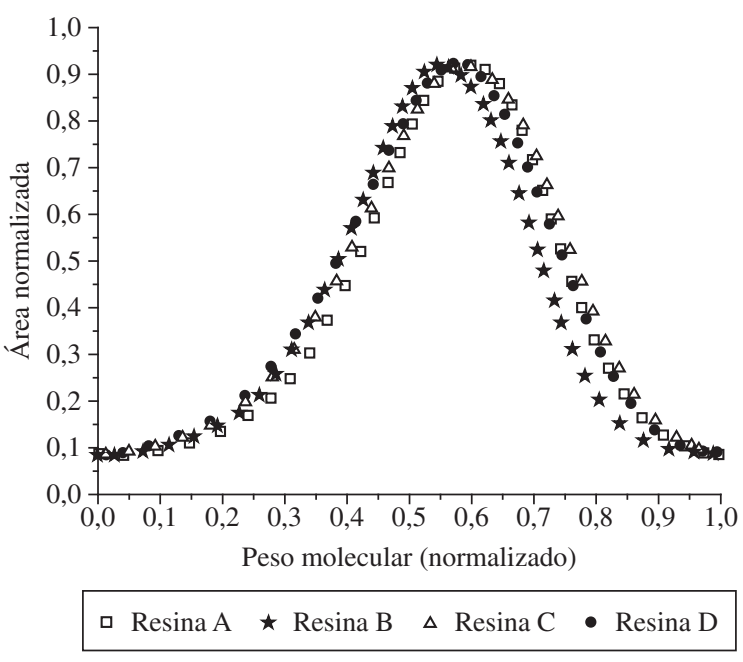

Figura 1. Distribuição de massas molares das resinas A, B, C e D.

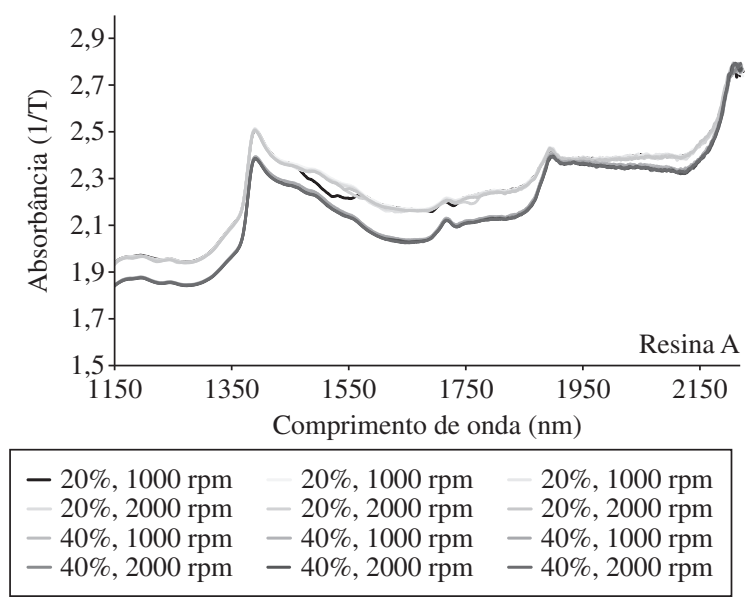

(a)

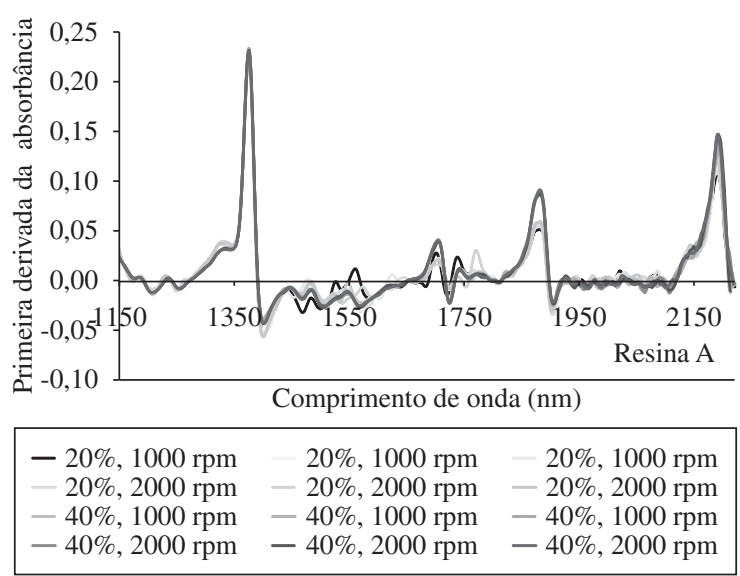

(b)

Figura 2. Espectros de NIRS da resina A. a) Bruto; e b) $1^{\text {a }}$ derivada.

tração de iniciador, como ilustrado na Figura 3a. Este resultado pode ser facilmente compreendido em termos da cinética clássica de reações radicalares, que mostra que as taxas de reação são proporcionais à raiz quadrada da composição do iniciador.

A massa molar do PVC é determinada fundamentalmente pela temperatura da reação, como ilustrado na Figura 3b. Isto comprova que o controle da estrutura molecular é devido à etapa de transferência de cadeia ao monômero. Quanto maior a temperatura, menor o valor $\mathrm{K}$ da resina. Por isso, na ausência de agentes de transferência de cadeia, a massa molar do PVC é controlada somente pela temperatura de reação, não sendo influenciada por outras variáveis, como a concentração de iniciadores.

A BD não foi afetada nem pela temperatura nem pela concentração de iniciador, como ilustrado na Figura 3c, d, mostrando que estes fatores não influenciam ou influenciam pouco o fenômeno de quebra-coalescência das gotas para a faixa de temperatura dos experimentos $\left(8^{\circ} \mathrm{C}\right)$. $\mathrm{O}$ fenômeno de quebra-coalescência é afetado basicamente pela velocidade de agitação e pelo tipo e quantidade de dispersantes no meio reacional. 


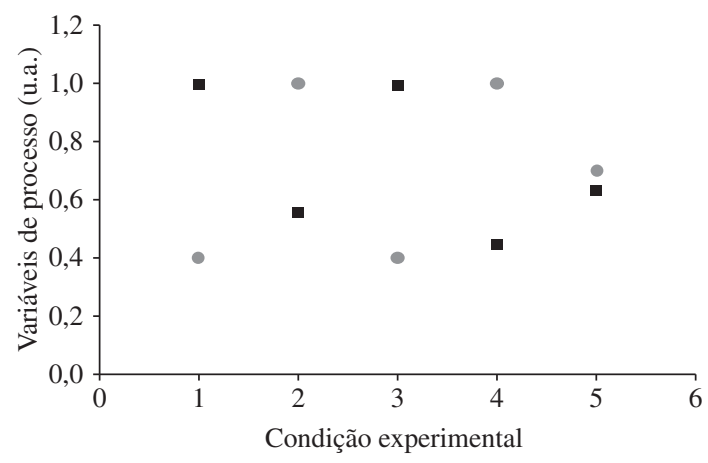

- Concentração de iniciador . Tempo de reação

(a)

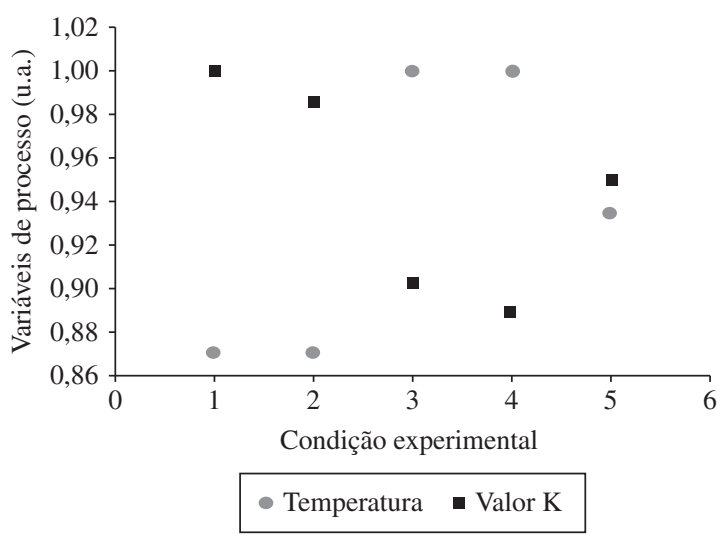

(b)

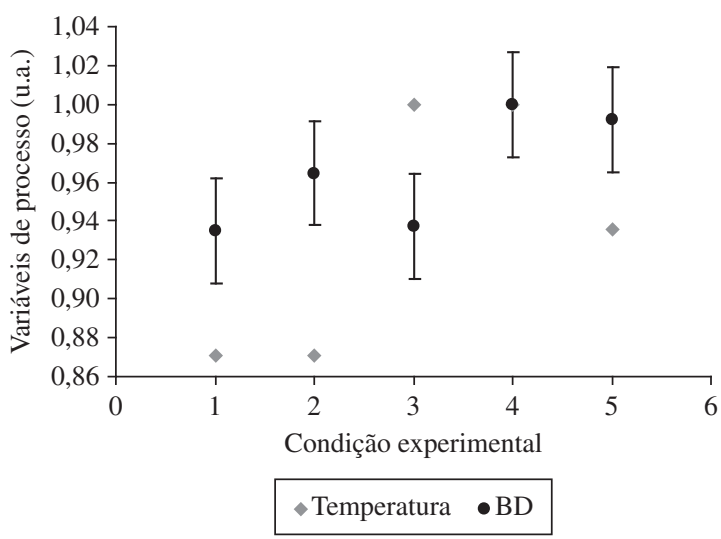

(c)

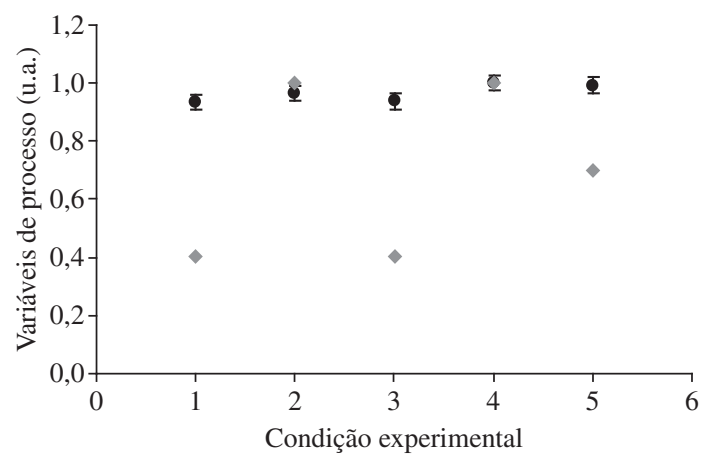

- BD Concentração iniciador

(d)

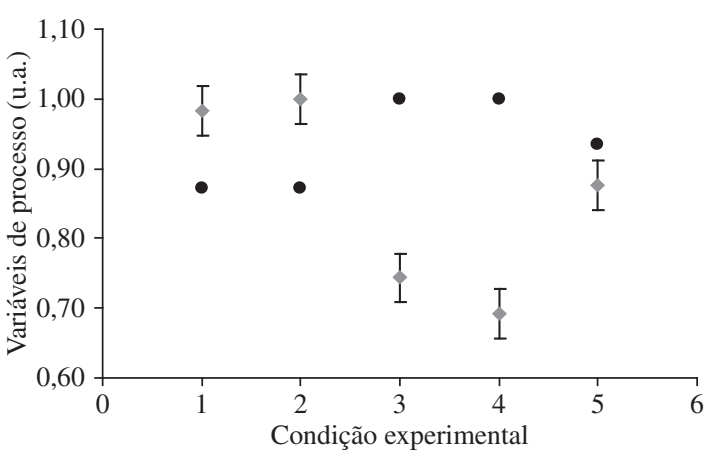

- Temperatura $\triangle \mathrm{CPA}$

(e)

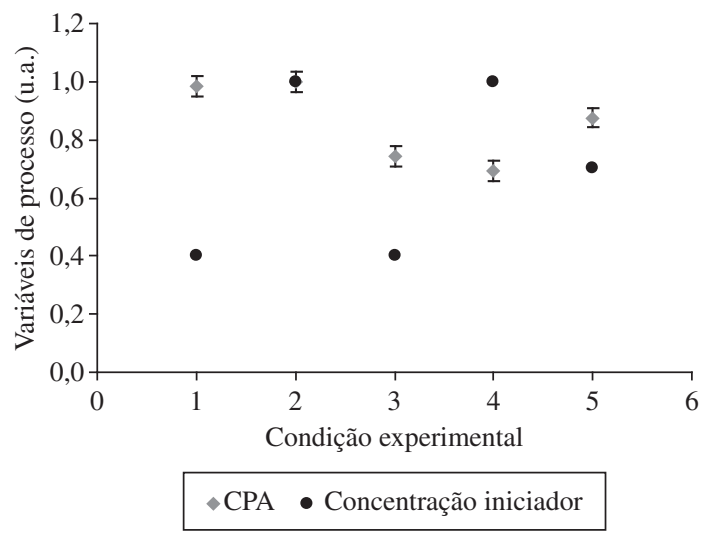

(f)

Figura 3. Avaliação das condicões experimentais. a) Influência da concentração de iniciador sobre o tempo de reação; b) influência da temperatura de reação sobre o valor K da resina; c) influência da temperatura de reação sobre a BD; d) influência da concentração de iniciador sobre a BD; e) influência da temperatura de reação o CPA; e f) influência da concentração de iniciador sobre o CPA.

A porosidade foi afetada pela temperatura de reação e pela concentração de iniciador, indicando o vínculo entre a porosidade e a cinética de polimerização, como ilustrado na Figura 3e, f. Em temperaturas mais elevadas, as taxas de reação e coalescência são maiores, o que favorece a aglomeração das partículas de PVC, fazendo com que o CPA seja menor.

Mostrou-se pela primeira vez que o acoplamento entre a cinética da polimerização e o fenômeno de quebra-coales- cência ocorre principalmente por meio das mudanças das propriedades físicas (viscosidade e tensão interfacial). No caso da polimerização do PVC, esse acoplamento é fraco, uma vez que o polímero precipita dentro das gotas de monômero logo após a formação. Por isso, não surpreende que a modificação da temperatura e da concentração de iniciador (variáveis que modificam principalmente as taxas de reação) exerça pouca influência sobre a BD. Com relação ao CPA, ele reflete principalmente a porosidade das partículas, que é 
grandemente influenciada pela estrutura dos microdomínios formados durante a precipitação do PVC. Portanto, o CPA depende muito mais fortemente das variáveis cinéticas (que modificam o tamanho dos grãos formados no interior das partículas) do que a BD. Os dados obtidos parecem refletir esse comportamento.

Analisando os espectros como ilustrado nas Figuras 4a, b verifica-se que eles mudam com o tempo de reação na região de 1600 a $1750 \mathrm{~nm}$, região que tem sensibilidade ao "tamanho de partícula" segundo Santos et al. ${ }^{[4,5]}$. Como as bateladas foram realizadas em condições bastante distintas, como mostrado nas Tabelas 2 e 3, isto é um indicativo de que a morfologia da partícula de PVC está mudando com o avanço da reação de polimerização e com as variáveis de operação como veremos posteriormente. As mudanças dos espectros estão associadas principalmente a fenômenos de espalhamento de
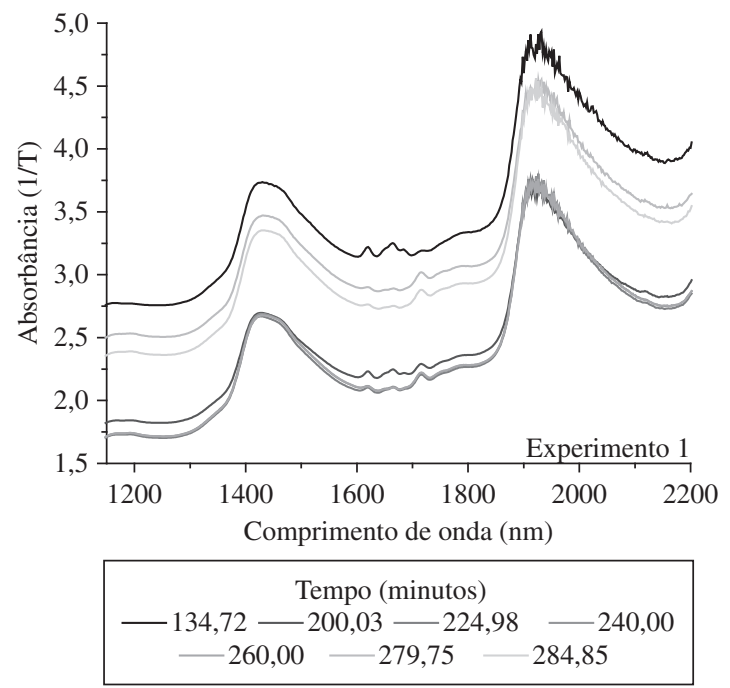

(a)

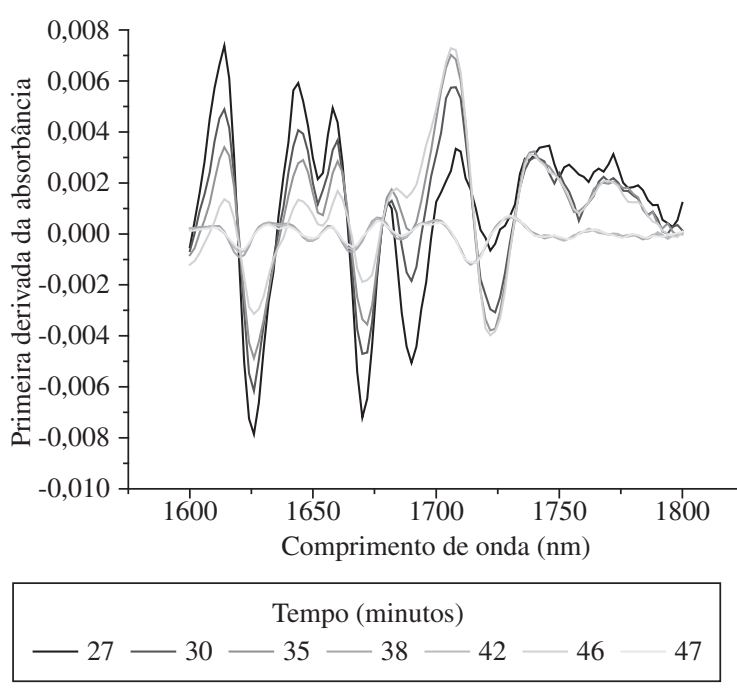

(b)

Figura 4. Espectros do experimento 1. a) Espectro bruto; e b) primeira derivada. Aumento na região de 1600 a $1800 \mathrm{~nm}$.
Tabela 2. Valor das variáveis nos três níveis.

\begin{tabular}{lcccc}
\hline \multicolumn{1}{c}{ Variável } & Unidade & Nível 1 & Nível 2 & Nível 3 \\
\hline $\mathrm{X}_{1}$ - Velocidade de & $\mathrm{rpm}$ & $\mathrm{X}$ & $1,58 \mathrm{X}$ & $2,16 \mathrm{X}$ \\
$\begin{array}{l}\text { agitação } \omega \\
\mathrm{X}_{2}-\text { Temperatura }\end{array}$ & ${ }^{\circ} \mathrm{C}$ & $\mathrm{Y}$ & $1,05 \mathrm{Y}$ & $1,1 \mathrm{Y}$ \\
$\mathrm{X}_{3}$ - MVC & $\mathrm{g}$ & $\mathrm{Z}$ & $1,25 \mathrm{Z}$ & $1,5 \mathrm{Z}$ \\
$\mathrm{X}_{4}$ - Produto A & $\mathrm{phm}$ & $\mathrm{K}$ & $1,06 \mathrm{~K}$ & $1,12 \mathrm{~K}$ \\
$\mathrm{X}_{5}$ - Produto B & $\mathrm{phm}$ & $\mathrm{L}$ & $1,11 \mathrm{~L}$ & $1,22 \mathrm{~L}$ \\
$\mathrm{X}_{6}$ - Produto C & $\mathrm{phm}$ & $\mathrm{M}$ & $2 \mathrm{M}$ & $3 \mathrm{M}$ \\
\hline
\end{tabular}

luz. Elas também podem ser geradas por outros fatores, que não a morfologia.

Analisando os espectros de MVC puro (na ausência de água) da Figura 5, verifica-se que na região de $1600 \mathrm{~nm} \mathrm{o}$ MVC tem um sinal de absorbância muito intenso, mostrando que nesta região há informação relativa à ligação dupla do MVC e que, por isso, pode se avaliar a possibilidade de calibrar os espectros NIRS para predição da conversão. Comparando as Figuras 4 e 5, observa-se que o sinal da insaturação vinílica fica bastante atenuado, em função do consumo de MVC e, principalmente, do espalhamento provocado pelas gotas dispersas.

Analisando os espectros finais de primeira derivada de diferentes bateladas, mostrados na Figura 6a, é possível afirmar que na região de $1600 \mathrm{~nm}$ não existe apenas informação da ligação dupla. Observa-se que os espectros são distintos entre si e no final da reação onde a quantidade de MVC presente no meio reacional é muito pequena, eles enxergam diferentes resinas de PVC, ou seja, diferentes morfologias. Isto indica que é possível ver características morfológicas, como será mostrado adiante.

Analisando os espectros finais de primeira derivada de bateladas com mesma carga de sólidos da Figura $6 \mathrm{~b}$ (Experimentos 1, 2, 3, 4, 9, 12, 13 e 18 da Tabela 3) verifica-se que os espectros são diferentes entre si, o que comprova que não é somente a carga que está alterando a sua forma. Isto indica que é possível ver características morfológicas, como será mostrado adiante.

Com o objetivo de construir um modelo de calibração que relacione parâmetros morfológicos da resina de PVC com espectros de infravermelho próximo, foram realizados experimentos para o levantamento dos dados morfológicos. O modelo desejado tem a forma dada pela Equação 1.

$$
P M=f(A, \lambda)
$$

onde PM está relacionado as propriedades morfológicas da resina de PVC (BD, CPA, D $)$, A é a absorbância e $\lambda$ é o comprimento de onda da radiação NIR.

Para obtenção dos dados morfológicos foram realizadas 17 polimerizações de MVC em suspensão. Utilizando as ferramentas do software VISION ${ }^{[6]}$ e o conjunto de dados de calibração, foi realizada uma série de testes com a técnica matemática padrão PLS (mínimos quadrados parciais) para o desenvolvimento dos modelos de calibração. Em função 
Tabela 3. Plano de Taguchi com 16 experimentos com réplicas no ponto central.

\begin{tabular}{|c|c|c|c|c|c|c|}
\hline \multirow[t]{2}{*}{ Experimento } & $\omega$ & Temperatura & MVC & Produto A & Produto B & Produto C \\
\hline & $X_{1}$ & $\mathbf{X}_{2}$ & $\mathbf{X}_{3}$ & $\mathbf{X}_{4}$ & $X_{5}$ & $X_{6}$ \\
\hline 1 & -1 & -1 & +1 & +1 & +1 & +1 \\
\hline 2 & -1 & -1 & +1 & -1 & -1 & +1 \\
\hline 3 & -1 & +1 & +1 & +1 & -1 & -1 \\
\hline 4 & -1 & +1 & +1 & -1 & +1 & -1 \\
\hline 5 & +1 & -1 & -1 & +1 & +1 & +1 \\
\hline 6 & +1 & -1 & -1 & -1 & -1 & +1 \\
\hline 7 & -1 & +1 & -1 & +1 & +1 & +1 \\
\hline 8 & -1 & +1 & -1 & -1 & -1 & +1 \\
\hline 9 & +1 & -1 & +1 & -1 & +1 & -1 \\
\hline 10 & +1 & +1 & -1 & +1 & -1 & -1 \\
\hline 11 & +1 & +1 & -1 & -1 & +1 & -1 \\
\hline 12 & +1 & +1 & +1 & +1 & +1 & +1 \\
\hline 13 & +1 & +1 & +1 & -1 & -1 & +1 \\
\hline 14 & 0 & 0 & 0 & 0 & 0 & 0 \\
\hline 15 & -1 & -1 & -1 & -1 & +1 & -1 \\
\hline 16 & -1 & -1 & -1 & +1 & -1 & -1 \\
\hline 17 & +1 & -1 & +1 & +1 & -1 & -1 \\
\hline
\end{tabular}

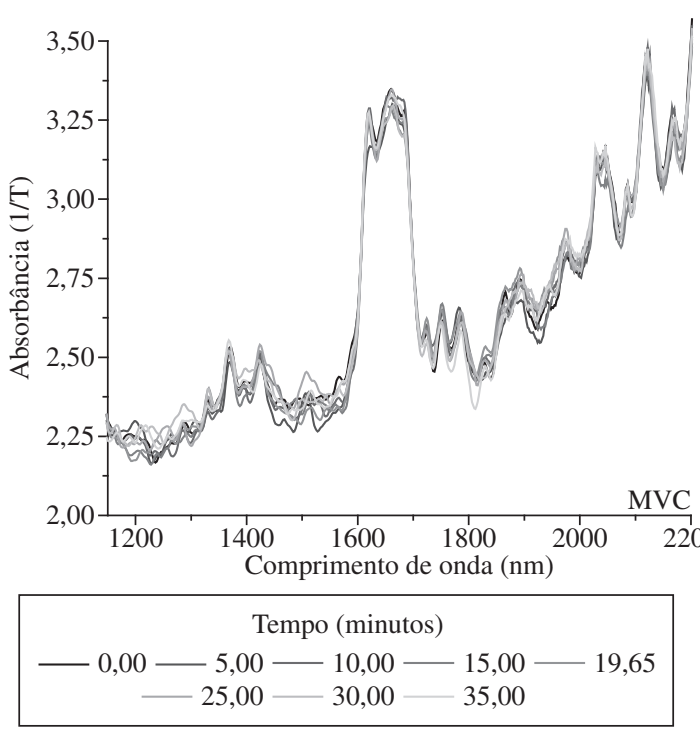

Figura 5. Espectro bruto do MVC.

do menor erro quadrático foram utilizados oito fatores para a construção do modelo de calibração para BD e dez fatores para o CPA e o Dp. Salienta-se que podem ser utilizados modelos de calibração com 5 e 8 fatores respectivamente para a BD, para o CPA e para o Dp, o que aumenta a robustez dos modelos desenvolvidos. A diferença no número de dados experimentais utilizados para construção dos modelos de calibração para as variáveis morfológicas foi devida à retirada dos valores espúrios do conjunto experimental, detectados durante o processo de calibração.

A calibração foi feita somente com os dados finais das bateladas, porque não faz sentido medir $\mathrm{BD}, \mathrm{CPA}, \mathrm{D}_{\mathrm{p}}$, para tempos menores que o tempo final das mesmas. Na Figura $7 \mathrm{e}$ Tabela 4 são mostrados os resultados obtidos nesta fase.

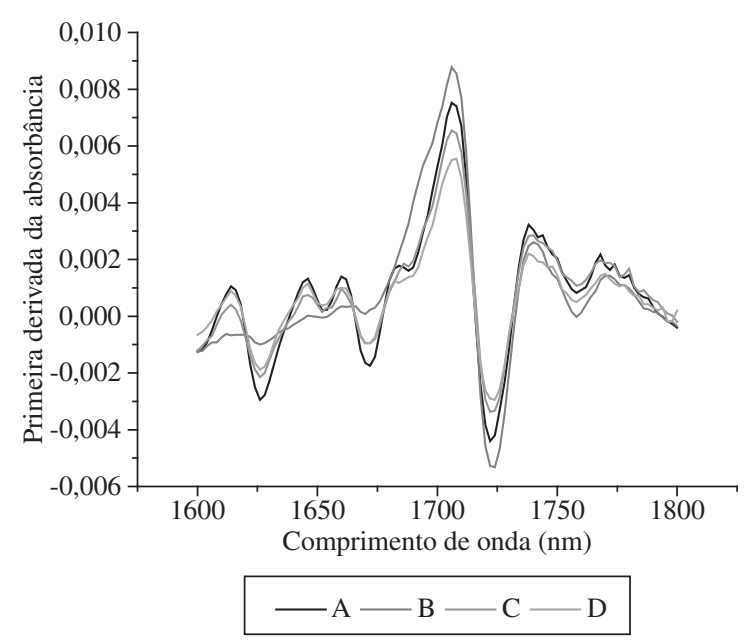

(a)

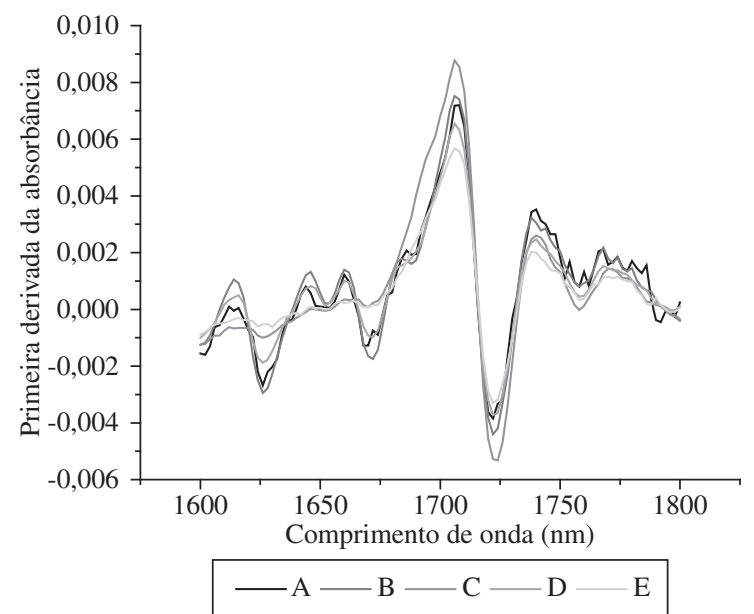

(b)

Figura 6. Derivada dos espectros finais de reação a) diferentes receitas; e b) mesma carga de sólidos. 


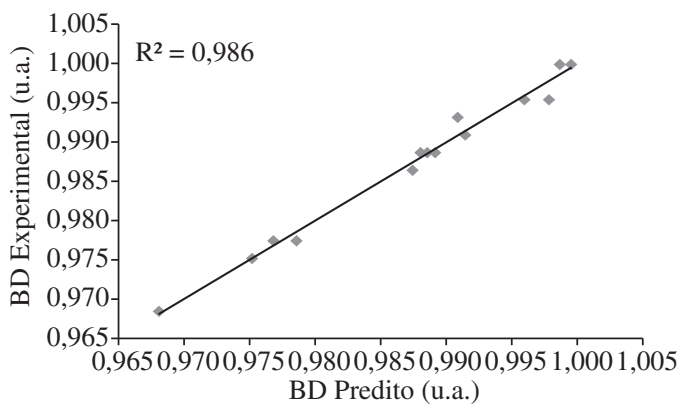

• BD Experimental _ Regressão linear

(a)

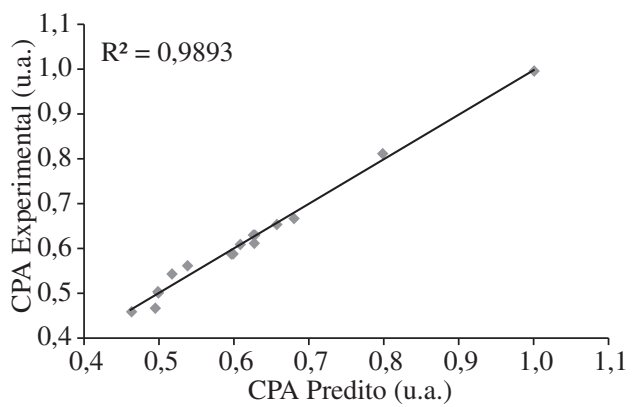

- CPA Experimental — Regressão linear

(b)

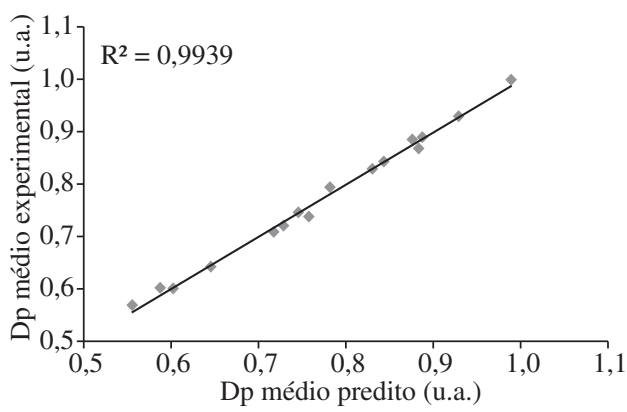

- Dp Experimental — Regressão linear

(c)

Figura 7. Predições do modelo de regressão. a) BD; b) CPA; e c) Dp médio.

Tabela 4. Fatores da curva de calibração para os parâmetros morfológicos do PVC.

\begin{tabular}{cccl}
\hline \multirow{2}{*}{ Fatores } & \multicolumn{3}{c}{ Erro quadrático $\mathbf{R}^{2}$} \\
\cline { 2 - 4 } & BD & CPA & \multicolumn{1}{c}{ Dp } \\
\hline 1 & 0,3589 & 0,189 & 0,44 \\
2 & 0,6225 & 0,243 & 0,528 \\
3 & 0,7063 & 0,302 & 0,607 \\
4 & 0,7902 & 0,435 & 0,726 \\
5 & 0,9407 & 0,684 & 0,822 \\
6 & 0,9612 & 0,811 & 0,856 \\
7 & 0,98 & 0,868 & 0,903 \\
8 & 0,986 & 0,945 & 0,946 \\
9 & - & 0,972 & 0,984 \\
10 & - & 0,989 & 0,994 \\
\hline
\end{tabular}

As correlações obtidas para inferência da BD e CPA da resina foram excelentes e capazes de representar os dados experimentais. Analisando os espectros como ilustrado na Figura 8 para os experimentos 6 e 8 da Tabela 3, verificase a qualidade das predições realizadas para a BD e CPA ao longo de toda a batelada com o modelo de calibração obtido. Como o intervalo de aquisição de cada espectro médio nas reações de polimerização monitoradas por NIR foi de 300 segundos, quanto maior for o número de espectros coletados, maior será o tempo de reação da batelada. Uma explicação para o ruído inicial pode ser que não tenha sido atingido o equilíbrio dinâmico entre quebra e coalescência das gotas de monômero no início da polimerização. As evoluções dinâmicas das variáveis BD e CPA são completa-
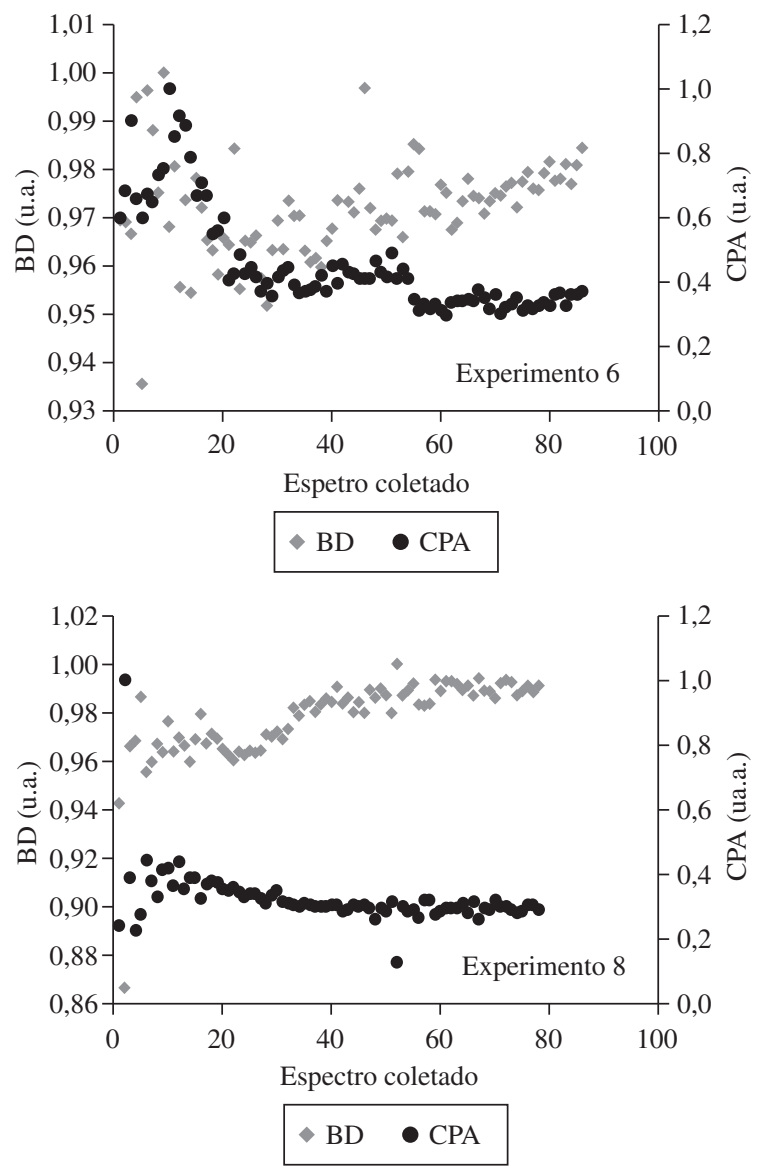

Figura 8. Trajetórias dinâmicas preditivas para BD e CPA obtidas com os modelos de calibração desenvolvidos. 
mente preditivas, uma vez que apenas os espectros finais foram de fato usados para calibração dos modelos. Estas figuras apresentam resultados bastante inovadores, pois não há relatos na literatura aberta do monitoramento dessas propriedades em linha e em tempo real com o uso de uma sonda NIR.

Os resultados obtidos indicam claramente que a técnica NIRS pode ser utilizada com sucesso para monitorar propriedades ligadas à morfologia das partículas de PVC. Esta conclusão está baseada no fato de que as previsões espectrais flutuam em torno de valores médios bem estabelecidos e, na maior parte das vezes, na faixa de precisão experimental. Portanto, o espalhamento relativo dos dados não deve ser excessivamente valorizado, já que as escalas são sempre estreitas. Se as predições oriundas da análise dinâmica de CPA e BD são comparadas entre si, ficam claras as mudanças de grade e das características finais da resina, detectadas de forma pertinente pela técnica de NIRS.

A estabilidade relativa das medidas de NIRS ao longo de toda a trajetória experimental mostra que propriedades finais de interesse, como o BD e o CPA podem ser acompanhadas em linha durante o processo de polimerização.

Na Figura 9a são mostrados os valores de BD e CPA obtidos nas polimerizações realizadas em escala de bancada com mudança da velocidade de agitação durante a reação. Nota-se

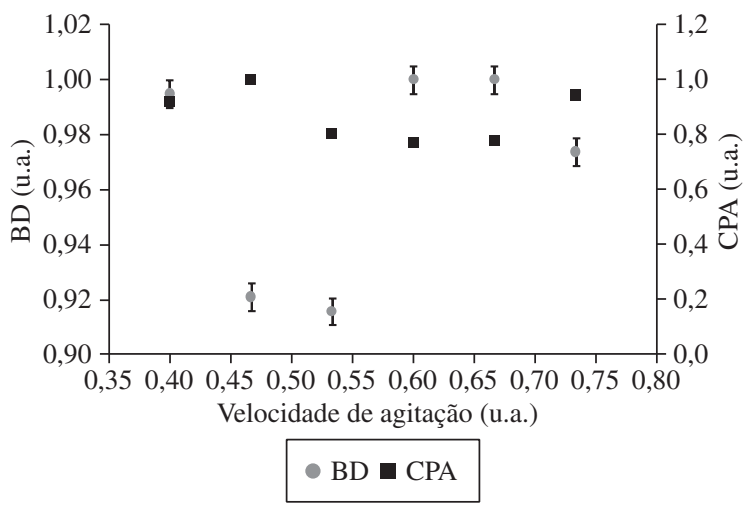

(a)

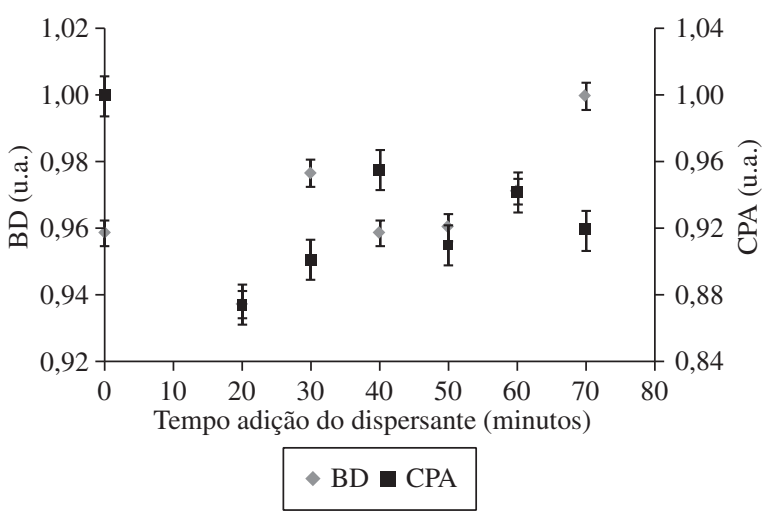

(b)

Figura 9. Valores de BD e CPA normalizados. a) Variação da velocidade de agitação; e b) adição de dispersantes. que estes parâmetros são alterados com o aumento da velocidade de agitação, mostrando que a velocidade de agitação pode ser manipulada para fins de controle das variáveis morfológicas do PVC.

Na Figura 10 são mostrados os valores de CPA e BD obtidos nos testes de adição de uma quantidade fixa de dispersante em diferentes tempos de reação. Os resultados su-
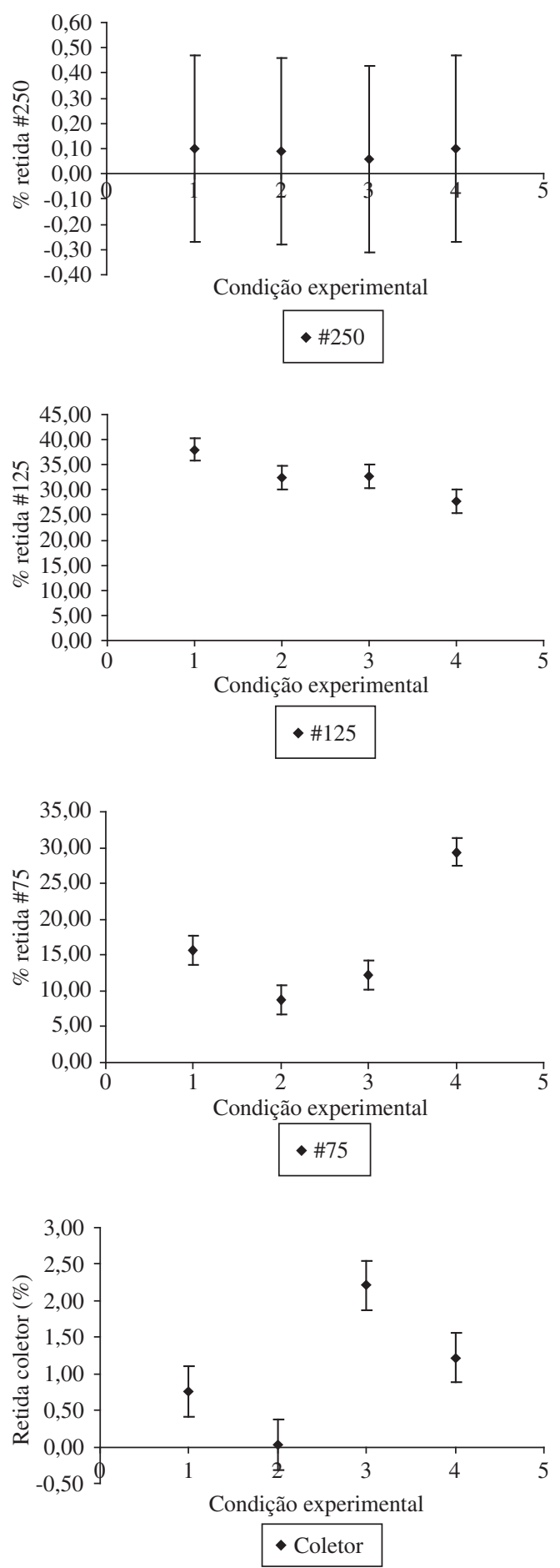

Figura 10. Distribuição de tamanhos de partícula de PVC vs. quantidade de dispersante adicionada com 20 minutos de reação. 

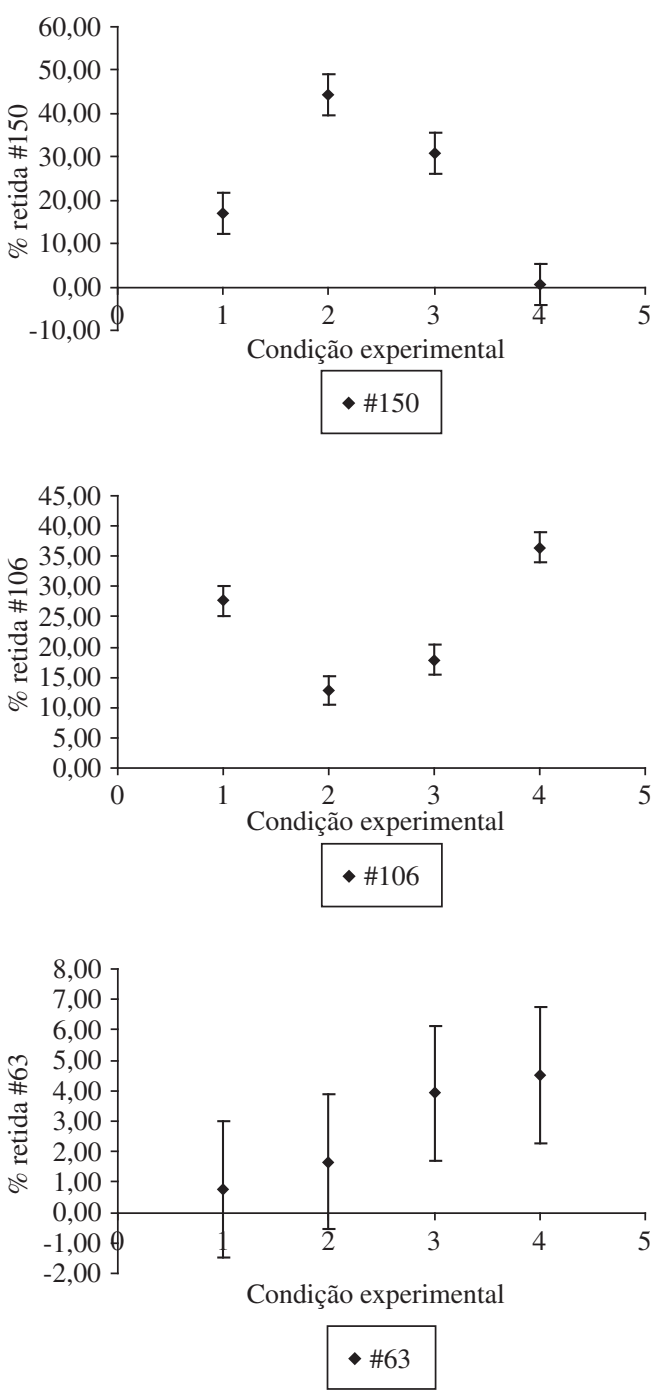

Figura 10. Continuação...

gerem que até 70 minutos de reação, para um tempo médio de reação de 250 minutos, pode-se perturbar o sistema pela adição de dispersantes ao meio reacional, com objetivo de mudar as trajetórias dinâmicas dos parâmetros morfológicos da resina de PVC. O tempo de adição de "0 minuto" é, na verdade, a batelada de referência (sem adição de dispersante).

Os resultados obtidos também mostram que é possível injetar quantidades crescentes de dispersante ao meio reacional para obtenção de resinas com distribuição de tamanhos de partícula distintas (Figura 10), embora nas malhas de \#250, \#125 e \#63 não sejam observadas mudanças.

Nas Figuras 11a, b são mostrados os valores do CPA e BD obtidos nas polimerizações com adição de quantidades de dispersantes crescentes durante a reação após 20 minutos do início do aquecimento. Conclui-se que é possível manipular a quantidade de dispersante dosada ao meio reacional para fins de controle das variáveis morfológicas do PVC.

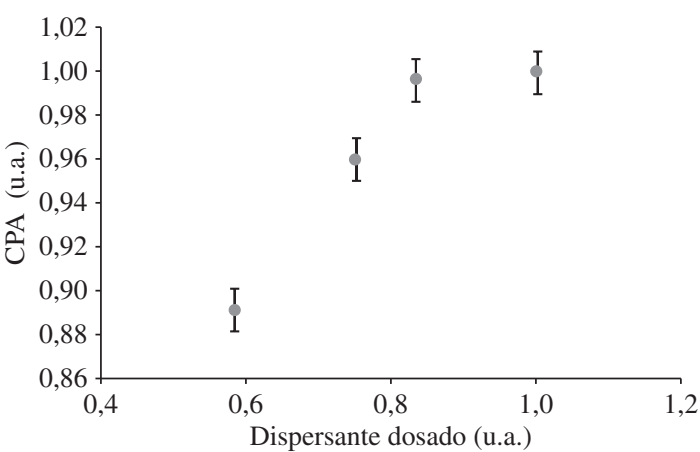

(a)

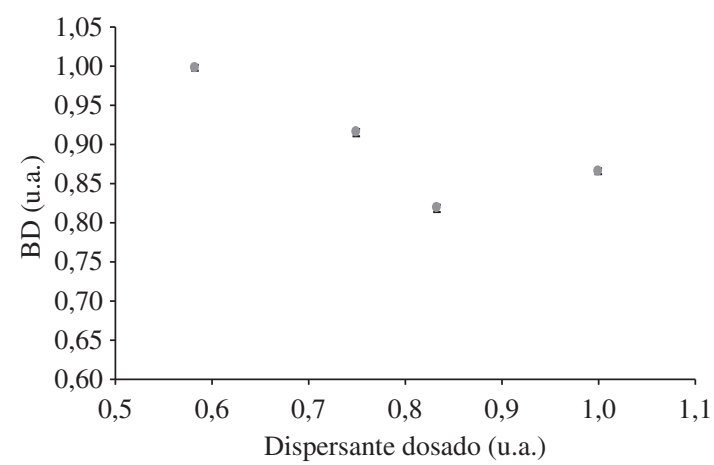

(b)

Figura 11. Avaliação da adição de dispersante. a) CPA normalizado; e b) BD normalizada.

\section{Conclusão}

Neste trabalho foi ilustrado o uso de espectroscopia do infravermelho próximo para fins de monitoramento em tempo real da polimerização em suspensão de MVC, buscando a predição e o acompanhamento de importantes propriedades morfológicas.

Mostrou-se pela primeira vez que é possível acompanhar, em tempo real, a evolução de propriedades morfológicas de resinas de PVC, como por exemplo, BD, CPA, DTP e Dp, por meio da construção de modelos de calibração empregados para estabelecer as correlações empíricas entre as variáveis medidas e os espectros de NIRS.

Evidenciou-se que a carga e a conversão das polimerizações afetam os espectros NIR. Mostrou-se também que o acoplamento entre a cinética de polimerização e o fenômeno de quebra-coalescência das gotas de monômero é fraco, uma vez que o polímero precipita nas gotas de monômero logo após a formação.

Com a possibilidade de predizer a evolução dinâmica dos parâmetros morfológicos em tempo real, mostrou-se que é possível injetar dispersantes e variar a velocidade de agitação durante a reação para fins de controle das variáveis $\mathrm{BD}, \mathrm{CPA}$ e DTP do PVC, até que o ponto de identificação da partícula seja atingido. 


\section{Agradecimentos}

Os autores agradecem à Braskem S.A. através do programa NEO PVC (Núcleo de Estudos Orientados do PVC) e à FINEP pelo suporte técnico necessários ao desenvolvimento do trabalho.

\section{Referências Bibliográficas}

1. De Faria Jr., J. M. - Monitoramento in situ e em Tempo Real de Variáveis Morfológicas do PVC com o Uso de uma Sonda NIR. 2008: Tese de D.Sc., COPPE/UFRJ, Rio de Janeiro, RJ, Brasil.
2. Santos, A. F., Lenzi, M. K., Machado, F. \& Pinto, J. C. Polymer-Plastics Technology and Engineering, 44, p.1 (2005).

3. Kammona, O., Chatzi, E. G. \& Kiparissides, C. - J.M.S. REV. Macromol. Chem. Phys, C39, (1), p. 57 (1999).

4. Santos, A. F., Lima, E. L. \& Pinto, J. C. - Journal of Applied Polymer Science, 70, p. 1737 (1998).

5. Santos, A. F., Lima, E. L. \& Pinto, J. C. - Journal of Applied Polymer Science, 77, p. 453 (2000).

6. VISION 3.2, Reference Vision ${ }^{\circledR}$ Manual, Spectral Analysis Software for Windows, Foss NIRSystems Inc, USA (2004). 